Article

\title{
Simulation of Optical Nano-Manipulation with Metallic Single and Dual Probe Irradiated by Polarized Near-Field Laser
}

\author{
Genwang Wang ${ }^{1,2}$, Ye Ding ${ }^{1,2}$, Haotian Long ${ }^{1,2}$, Yanchao Guan ${ }^{1,2}$, Xiwen Lu ${ }^{1,2}$, Yang Wang ${ }^{1,2, *}$ \\ and Lijun Yang ${ }^{1,2, *}$
}

check for updates

Citation: Wang, G.; Ding, Y.; Long, H.; Guan, Y.; Lu, X.; Wang, Y.; Yang, L. Simulation of Optical Nano-Manipulation with Metallic Single and Dual Probe Irradiated by Polarized Near-Field Laser. Appl. Sci. 2022, 12, 815. https://doi.org/ 10.3390/app12020815

Academic Editor: Francesco Tornabene

Received: 19 December 2021

Accepted: 12 January 2022

Published: 13 January 2022

Publisher's Note: MDPI stays neutral with regard to jurisdictional claims in published maps and institutional affiliations.

Copyright: (C) 2022 by the authors. Licensee MDPI, Basel, Switzerland. This article is an open access article distributed under the terms and conditions of the Creative Commons Attribution (CC BY) license (https:// creativecommons.org/licenses/by/ $4.0 /)$.
1 State Key Laboratory of Robotics and Systems, Harbin Institute of Technology, Harbin 150001, China; nisker@163.com (G.W.); dy1992hit@hit.edu.cn (Y.D.); 13069882881@163.com (H.L.); guanyanchaoo@163.com (Y.G.); lu_xiwen@163.com (X.L.)

2 School of Mechatronics Engineering, Harbin Institute of Technology, Harbin 150001, China

* Correspondence: wyyh@hit.edu.cn (Y.W.); yljtj@hit.edu.cn (L.Y.)

\begin{abstract}
Nano-manipulation technology, as a kind of "bottom-up" tool, has exhibited an excellent capacity in the field of measurement and fabrication on the nanoscale. Although variety manipulation methods based on probes and microscopes were proposed and widely used due to locating and imaging with high resolution, the development of non-contacted schemes for these methods is still indispensable to operate small objects without damage. However, optical manipulation, especially near-field trapping, is a perfect candidate for establishing brilliant manipulation systems. This paper reports about simulations on the electric and force fields at the tips of metallic probes irradiated by polarized laser outputted coming from a scanning near-field optical microscope probe. Distributions of electric and force field at the tip of a probe have proven that the polarized laser can induce nanoscale evanescent fields with high intensity, which arouse effective force to move nanoparticles. Moreover, schemes with dual probes are also presented and discussed in this paper. Simulation results indicate that different combinations of metallic probes and polarized lasers will provide diverse near-field and corresponding optical force. With the suitable direction of probes and polarization direction, the dual probe exhibits higher trapping force and wider effective wavelength range than a single probe. So, these results give more novel and promising selections for realizing optical manipulation in experiments, so that distinguished multi-functional manipulation systems can be developed.
\end{abstract}

Keywords: nano-manipulation; optical tweezers; near-field; evanescent field; atomic force microscope; scanning near-field optical microscope

\section{Introduction}

Since xenon atoms were successfully positioned and assembled with atomic precision [1], nano-manipulation technology, as a kind of "bottom-up" tool, has attracted wide attention in the fields of nano-measurement, nano-fabrication, nano-optics, nano-robot, and so on [2-5]. Among various nano-manipulation methods, the most widespread is probe manipulation based on high-resolution microscopes, including atomic force microscope (AFM), scanning tunneling microscope (STM), scanning electron microscope (SEM), and transmission electron microscope (TEM) [6-9]. With images feedback of targets, these systems use probes, which are carried by manipulators with nanoscale resolution of movement, as end-effectors to push, pick, stretch, place, and bend nanomaterials. The manipulation mechanism of this method is mainly based on the control of mechanical forces (e.g., contact force, Van der Waals force, friction) between probes, objects, and substrates $[10,11]$. So, due to the convenience of locating and imaging, this method exhibits outstanding manipulation capacity, and enormous potential in the application of nanomaterials, like tuning electrical and optical properties, constructing nanostructure, and even fabricating nano-devices. For instance, nanoparticles (NPs) and nanowires (NWs) 
can be stacked together by a tweezer which consists of two individually actuated probes in AFM or SEM $[12,13]$. Two-dimensional materials were folded and unfolded precisely by the STM-based manipulation system to improve electrical and quantum properties [14]. The AFM-based manipulation also has the capacity to assemble and weld gold nanowires for fabricating nano-circuits [15]. However, the most of manipulation methods mentioned above are always performed by direct contact between nanomaterials and probes, so that the damage of both is inevitable during processing. In addition, it also limits the ability of manipulating small objects due to the size of contact tips. Hence, the further exploration of non-contact manipulation benefits from the development of state-of-the-art methods and systems.

Optical tweezers, presented by Ashkin, represent a revolutionary tool for manipulating nanomaterials due to non-contact optical trapping, with or without less damage [16]. It has also proven useful not only for trapping and spinning nanomaterials and cells, but also for significant applications in nano-devices so far [17-20]. To manipulate much smaller NPs, near-field (also named as evanescent field) optical tweezers, which are beyond the optical diffraction limit, provide a novel high-resolution scheme. However, the capacity of this is also restricted by the small optical forces induced by weak evanescent fields. Hence, variety enhancement solutions have been proposed sequentially. For example, Mandal presented a kind of silicon photonic crystal resonators to trap dielectric NPs ranging in size from 50 to $500 \mathrm{~nm}$. This method provided a several-orders-of-magnitude stronger force and an order of magnitude stiffer than other conventional near-field tweezers [21]. Zhang successfully fabricated plasmonic dipole antennas to enhance near-field, which were capable of trapping $10 \mathrm{~nm}$ metal NPs [22]. Similarly, Abhay also built optical traps, based on a structure of double nano-hole, with high stiffness when manipulating $20 \mathrm{~nm}$ polystyrene NPs [23]. Although these solutions have proven their excellent ability for steady trapping, the targeted and flexible manipulation of small objects is still limited by the fixed enhancement structures and corresponding laser source. The limitation creates a high barrier of application. Hence, optical manipulation using mobile probes, like fiber probes, scanning near-field optical microscope (SNOM) probes, AFM probes, and even customized probes, has appeared to break through the bottleneck. Keyi proved that nanometer scale particles could be trapped using a SNOM probe by calculations [24]. Berthelot engineered a single SNOM probe with a bowtie aperture at the extremity to realize near-field nano-tweezers, and achieved the movement of trapped $50 \mathrm{~nm}$ objects in water [25]. Liu demonstrated a scheme which produced an optical trap at the tip of a single AFM probe, illumined by a near-field from the SNOM probe [26,27]. This novel method can trap NPs with sizes down to $20 \mathrm{~nm}$. Moreover, simulations proved that the trapping force depended on the distance between two probes, an incident angle, an incident polarization direction, and the wavelength of the laser. So, this scheme not only shows promising trapping ability, but also the convenience of moving the target due to the AFM system with high-resolution imaging and locating. In addition, this combination of enhancement near-field and AFM system also provides promising ways to achieve more multiple and accurate manipulation, and extend the application in nano-fabrication [28]. However, the present simulations and experiments of this scheme still mainly focus on laser-irradiated single probes in the AFM system. Thus, due to the development of a microscope-based manipulation system with multi-probes, a deeper insight into optical manipulation with different configurations of probes irradiated by near-field laser is promising and urgent for future application.

This paper presents schemes of metallic single and dual probes irradiated by a polarized laser coming from a SNOM probe, and calculates near-fields and corresponding optical forces around tips and an NP. The effect of laser wavelength, polarization direction and probe configurations on near-field was investigated via the three-dimensional finite difference time domain method (3D FDTD) to obtain effective optical traps. The calculation results demonstrate that the intensity and location of enhanced near-field heavily depend on the polarization direction for the different configuration of probes. Furthermore, optical 
forces applied on a silver NP were calculated by a Maxwell stress tensor. A single AFM probe was proven to induce a stable optical trap when the laser source polarized along the axis of the probe. Moreover, the dual probe also exhibits a more promising capacity of nano-manipulation, with a higher force and a wider range of the effective wavelength of the laser than the single probe. So, this work presents novel and more outstanding strategies for manipulating nanomaterial by combing metallic tips and a polarized near-field laser.

\section{Methods}

The simulations of near-field nano-manipulation were preformed based on 3D FDTD. Figure 1 shows the configurations of single probe and dual probe irradiated by polarized near-field laser coming from an SNOM probe. According to commercial probes, the model of the SNOM probe consists of the waveguide region and tip region. Both regions have a $\mathrm{SiO}_{2}$ core and an aluminum shell with thickness $h=80 \mathrm{~nm}$. The maximum and minimum diameters of $\mathrm{SiO}_{2}$ core in tip region are $\phi_{1}=700 \mathrm{~nm}$ and $\phi_{2}=200 \mathrm{~nm}$, and the length is $L=600 \mathrm{~nm}$. Likewise, the model of an AFM probe was simplified to a gold cone with angle $\theta=36^{\circ}$ and a spherical tip (the radius is set to $10 \mathrm{~nm}$ ). According to previous simulations, the enhancement of near-field and trapping force are stronger when the incident angle is $90^{\circ}$, with lower distance between the AFM and SNOM probes [26]. So, considering experimental feasibility, the incident angle and distance along $x$ direction of two probes were set to $90^{\circ}$ and $100 \mathrm{~nm}$, respectively. For dual probes, two configurations with angles $90^{\circ}$ and $180^{\circ}$ of axes of two AFM probes in $x z$ plane, named as orthogonal dual probes (ODPs) and coaxial dual probes (CDPs) respectively, were simulated in this paper, as shown in Figure 1c,d. A silver NP (the diameter is $d$ ) was located under the AFM tip with distances $D_{1}$ (single probe) or $D_{2}$ (dual probe, the distance between two tips was marked as $D_{3}$ ) to study the near-field enhancement and trapping force. Vertical polarized (VP, along axis $z$ ) and horizon polarized (HP, along axis $y$ ) lasers were coupled with the SNOM probe. In consideration of calculation efficiency, the incident laser was set to a normalized signal, as Figure 1e showed. Since the maximum amplitude was 1, the electric field intensity $E$ and trapping force $F$ calculated in this paper were both relative values. In this paper, relative values are enough to discuss the influence of the polarized laser and the configurations of probes on the trapping ability. Wavelengths ranging from $300 \mathrm{~nm}-1200 \mathrm{~nm}$ were adopted to explore the effect on manipulation. For global FDTD calculations, the simulation temperature was $300 \mathrm{~K}$. The size of the simulation area was $2 \mu \mathrm{m}(x) \times 1.1 \mu \mathrm{m}(y) \times 1.1 \mu \mathrm{m}(z)$ with mesh size $2 \mathrm{~nm}$, as the origin dash lines showed in Figure 1a,b. Moreover, to guarantee calculation accuracy, the area of AFM tips and NP (red dash lines) had a fine mesh with a $1 \mathrm{~nm}$ resolution. A perfectly matched layer with a minimum layer number of 12 was used to avoid energy reflection in the boundary. The simulation time $300 \mathrm{fs}$ was more than enough to achieve desirable results.

To calculate the trapping forces, the method based on Maxwell stress tensor combined with FDTD was applied. Given that the volume $v$ and corresponding external boundary $S$ contain NP, the force exerted on the NP can be written as:

$$
\boldsymbol{F}=\int_{v} \boldsymbol{f} \cdot d v=\oint_{S} \stackrel{\leftrightarrow}{\boldsymbol{T}} \cdot \boldsymbol{n} d S-\varepsilon_{0} \mu_{0} \int_{v} \frac{\partial S}{\partial t} d v
$$

where $S$ is Poynting vector. Here, for static magnetic field, $\partial S / \partial t=0 . \stackrel{\leftrightarrow}{T}$ is Maxwell stress tensor, which was defined as

$$
\stackrel{\leftrightarrow}{\boldsymbol{T}}=\varepsilon_{0}(\boldsymbol{E} \boldsymbol{E})-\frac{\varepsilon_{0}}{2}\left(\stackrel{\leftrightarrow}{I} \boldsymbol{E}^{2}\right)+\frac{1}{\mu_{0}}(\boldsymbol{B B})-\frac{1}{2 \mu_{0}}\left(\stackrel{\leftrightarrow}{I} \boldsymbol{B}^{2}\right)
$$

where $\varepsilon_{0}$ and $\mu_{0}$ are vacuum electric constant and magnetic constant, respectively. $\stackrel{\leftrightarrow}{I}$ is Kronecker's delta. $\boldsymbol{E}$ and $\boldsymbol{B}$ are electric and magnetic field respectively, which can be 
calculated by FDTD. So, Maxwell stress tensor and FDTD can be used to calculate the optical forces around NPs.

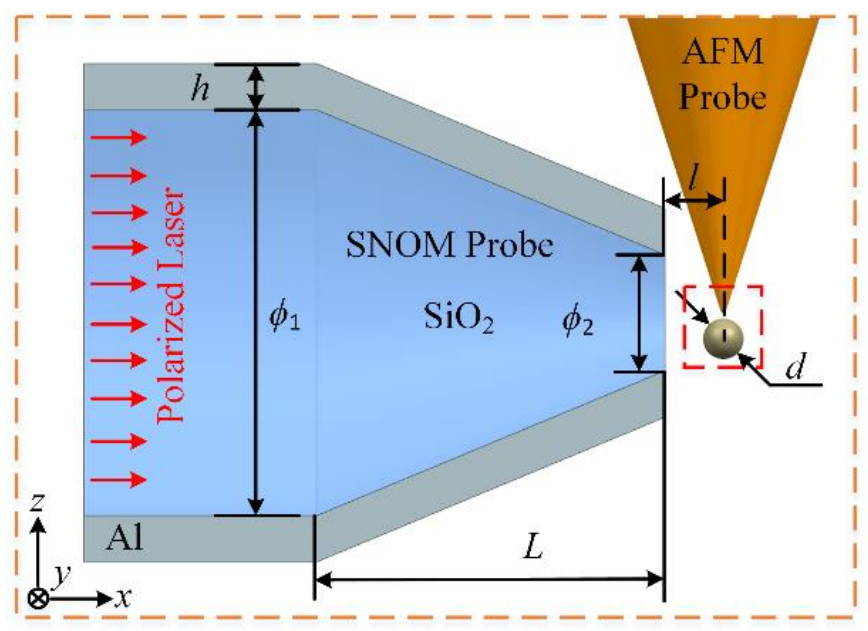

(a)

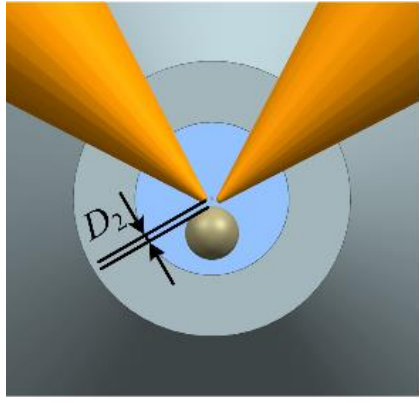

(c)

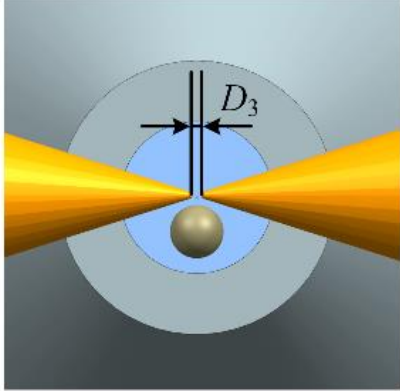

(d)

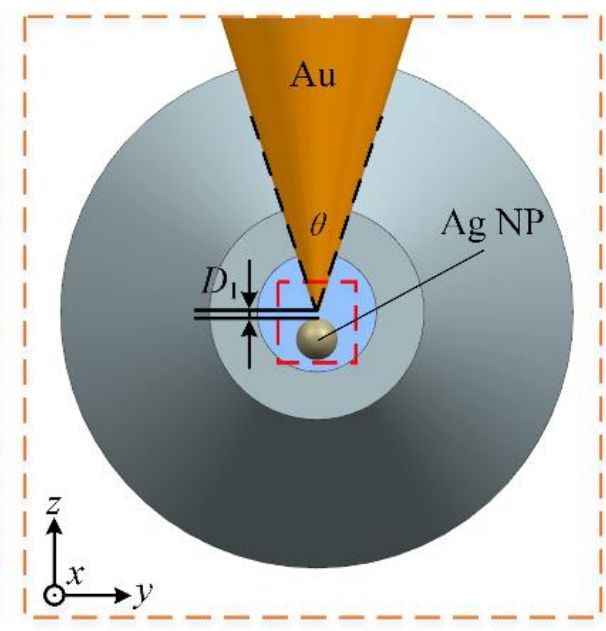

(b)

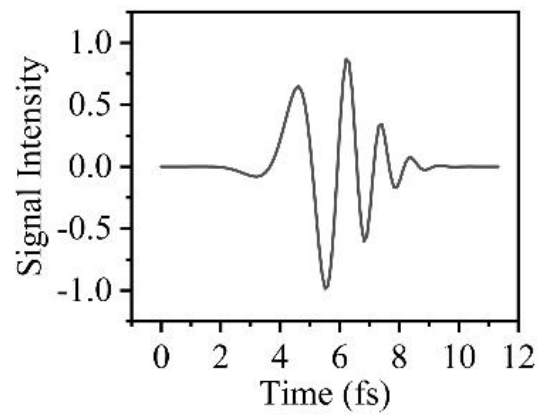

(e)

Figure 1. Configurations of single probe and dual probe irradiated by polarized near-field laser coming from a SNOM probe. Front view (a) and slide view (b) of single probe; Dual probes with axis angles $90^{\circ}$ (c) and $180^{\circ}$ (d); (e) Time signal of normalized laser.

\section{Results and Discussion}

\subsection{Distribution of Electric Field around Tip without NP}

According to the expression of the Maxwell stress tensor, before calculating the trapping force applied on an NP, the distribution of the electric field induced by polarized near-field laser around the AFM probe tips should be lucubrated. Figure 2a shows the distributions of electric field in the $x$-z plane, which was produced by the VP laser coming from a SNOM probe. The incident laser propagated in the $\mathrm{SiO}_{2}$ core and was reflected inward by the Al shell of the SNOM probe, so that the light formed an evanescent field along $x$ direction and then irradiated the tip of AFM probe through the output aperture. Hence, an enhancement field with size down to subwavelength was excited due to the localized-mode surface plasma at the surface of metallic tip. This small, enhanced field evanescent field is essential for manipulating NP. Although there are some enhancement fields at the edge of the output aperture and the cover shell of SNOM probes, the intensities of these are relatively lower and far from NPs. So, the effects of these enhancement fields on the NP can be ignored. To gain deep insight into the influence of the wavelength and polarization direction of laser on field enhancement, the electric intensities of the point under the probe tip are calculated, as in Figure $2 \mathrm{~b}$. According to the results, the VP laser induces particularly stronger enhancement at the end of the probe tip compared to the $\mathrm{HP}$ laser. The highest intensity is $\sim 8.7 \mathrm{~V} / \mathrm{m}$ for a wavelength around $717 \mathrm{~nm}$. Although there is a peak at $511 \mathrm{~nm}$ for the HP laser, the intensity is just $\sim 0.5 \mathrm{~V} / \mathrm{m}$. Figure $2 \mathrm{c}, \mathrm{d}$ 
presents the distribution of electric field in $y-z$ plane excited by the VP and HP laser with a wavelength of $717 \mathrm{~nm}$. Figure 2e,f also gives the corresponding line distributions of the electric field along the $y$-axis and $x$-axis, respectively. The white dash lines in Figure $2 \mathrm{c}, \mathrm{d}$ show the line paths. Illumined by the VP near-field laser, the AFM probe has a symmetrical evanescent field at the extremity, with the highest intensity of $\sim 11 \mathrm{~V} / \mathrm{m}$. Moreover, the intensity of this enhanced field decays exponentially along all directions, so that it has an effective area with a diameter lower than $80 \mathrm{~nm}$. Hence, this evanescent field provides a strong 3D gradient field to manipulate small NPs. If an NP is placed beneath the AFM probe, it could be trapped and moved towards the extreme intensity points of the gradient field. However, for the HP light, the enhancement exists at the two sides of the probe instead of at the end, as Figure $2 \mathrm{~d}$ showed. The maximum intensity is only $\sim 1.8 \mathrm{~V} / \mathrm{m}$. So, this weak and dispersive electric field cannot form a stable trap for NPs. Besides, there is also a small area with lower intensity at the end of the tip, see Figure 2e. The electric field induced by the laser with different wavelengths also has similar distributions (see Figure S1 in Supplementary Materials). So, comparing the two schemes, it is found that the intensity and location of near-field around the AFM probe tip heavily depend on the polarization direction of the incident laser rather than wavelength. If the polarization direction is parallel to the axial direction of the AFM probe, the near-field laser will induce an intensive nanoscale evanescent field, which is suitable for the stable trapping of NPs. However, when the polarization direction is perpendicular to the axial direction of the AFM probe, the evanescent exerted is incapable of desirable manipulation.

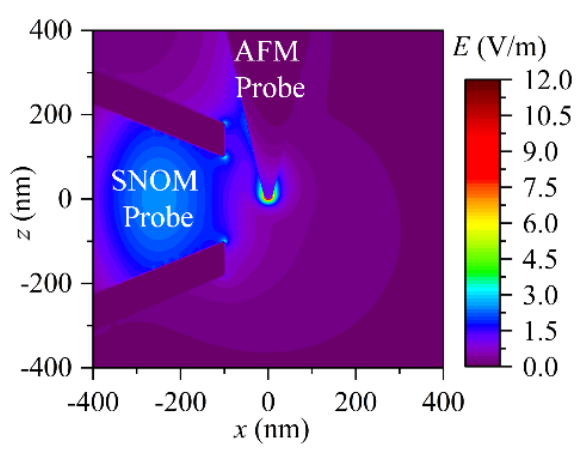

(a)

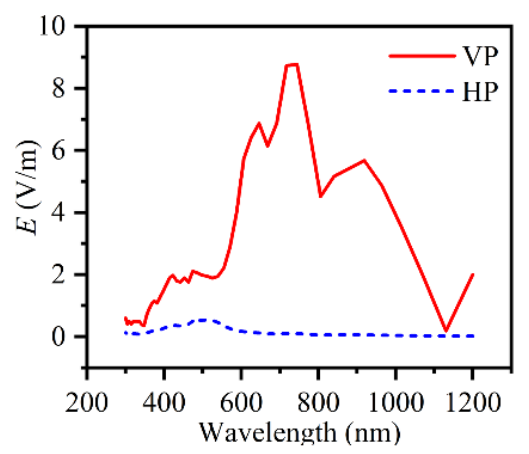

(b)

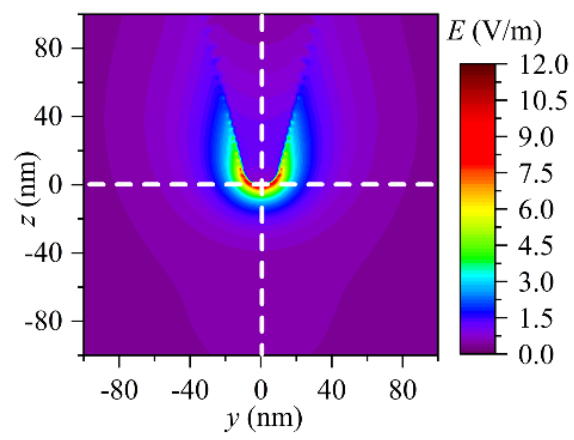

(c)

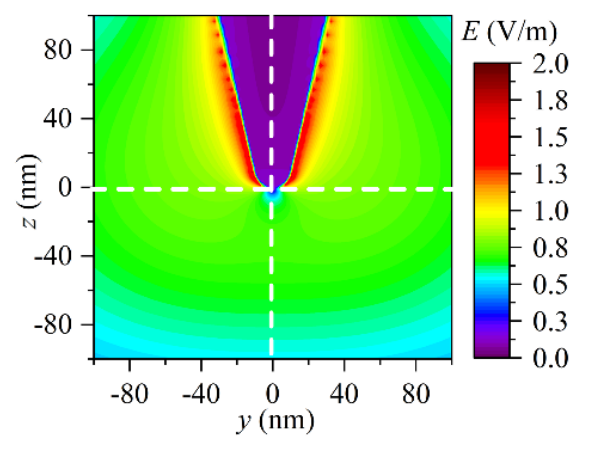

(d)

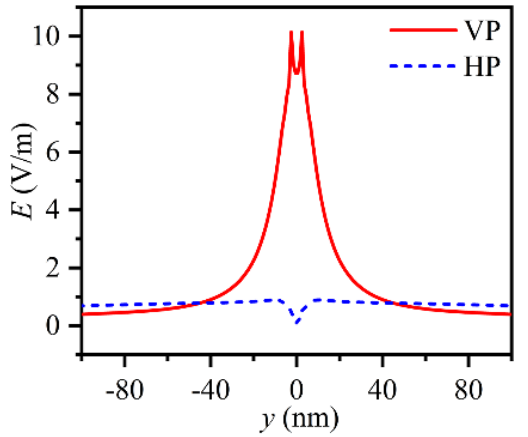

(e)

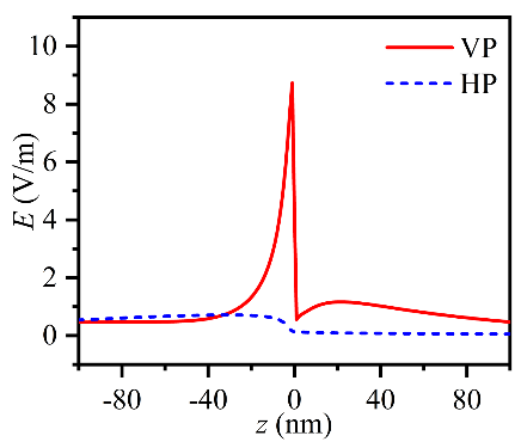

$(\mathbf{f})$

Figure 2. Single probe irradiated by polarized near-field laser coming from an SNOM probe. (a) Distributions of electric field around two probes induced by VP laser; (b) Electric intensities at the end of probe tip versus wavelengths of incident VP and HP laser. Distributions of electric fields in $y$-z plane induced by VP laser (c) and HP laser (d) with wavelength of $717 \mathrm{~nm}$; Corresponding distributions of electric field along $y$-axis (e) and $z$-axis (f).

Besides metallic tips, laser-irradiated structures with nano-gaps, like nano-antennas, nano-holes, and nano-bowties, are also advance methods to arouse and tune the evanescent 
field by adjusting the distance of the gap $[22,23,25]$. Considering that the probes of AFMbased or SEM-based manipulation systems can be located and moved with nanoscale or even sub-nanoscale resolution, nano-gaps can also be acquired by two metallic tips. So, in this paper, optical manipulating based on two configurations of ODPs and CDPs (see Figure 1c,d) was further simulated. Similar to single probe, the calculation results of the dual probe show that the wavelength of the laser mainly influences the maximum intensity of enhancement fields instead of the distribution. So, VP and HP lasers with a wavelength of $717 \mathrm{~nm}$ were taken as examples to explore the electric field around ODPs and CDPs. Likewise, the distances between two tips of all configurations were set to $D_{3}=10 \mathrm{~nm}$. The distributions are shown in Figure 3. For ODPs irradiated by the HP laser, the maximum intensity is $\sim 11.6 \mathrm{~V} / \mathrm{m}$, which is approximately equal to the single probe irradiated by the VP laser. According to the simulation results of the single probe irradiated by the VP and HP lasers in this paper and other previous works [26], the increase of the angle between the axis of the AFM probe and the polarized direction of the laser can lead to a decrease in enhancement intensity. Hence, if there was only one probe in the configuration of ODPs, the maximum intensity would be lower than the single probe irradiated by the parallel polarized laser (see Figure S2), because the probe has an angle with the polarized direction of the incident laser. However as the nano-gap between two nano-structures can improve the enhancement of the near-field [25], the small gap of the ODP helps to enhance the intensity of the optical field further. So, these negative and positive effects both result in the similar maximum intensity of the ODPs irradiated by HP laser comparing to the single probe irradiated VP laser, which also proves the capacity of dual probes to enhance the near-field. Moreover, the enhanced field, which is aroused between two metallic tips, has a larger high intensity area than the single probe. When the source changes to the VP laser, the enhanced field is divided into two parts located at the bottom of every probe, which is more like that of a single probe, as shown in Figure 3b. However, the intensity is lower (the maximum is $\sim 5.4 \mathrm{~V} / \mathrm{m}$ ). The decrease in intensity can also be attributed to the existing angles between the polarization direction of the laser and axial direction of AFM probes. If using CDPs, the HP laser induces an effective electric field for trapping a NP, since the axes of both probes are parallel to the laser polarization direction. The distribution of the electric field in Figure $3 \mathrm{c}$ also demonstrates that this configuration can provide the strongest enhancement and corresponding largest area with higher intensity (maximum: $\sim 18.4 \mathrm{~V} / \mathrm{m}$ ) than the others. However, the VP laser cannot produce a trap for manipulation, due to the dispersive electric field presented in Figure 3d. So, according to the electric distribution, three configurations, including ODPs irradiated by HP and VP lasers and CDPs by the HP laser, are suitable candidates for stable manipulation.

Since the size of the nano-gap has a significant effect on the enhancement, the electric fields of three desirable configurations with different probe distances $D_{3}$ were calculated. Figure 4 provides detailed distributions along the $y$ direction (the locations in $z$ direction are the same as for the white dash lines in Figure 3). For the HP laser, although the intensities of the enhancement field around ODPs and CDPs with small distances are higher than for the VP laser, they quickly decay as the distance increases. This phenomenon is consistent with previous works on the optical enhancement using nano-gaps [25]. So, it can be concluded that the conditions of forming desirable fields in these two schemes are stricter. However, despite relatively lower intensity, ODPs with different tip distances irradiated by the HP laser give a stable enhancement because of the separated two evanescent fields, as shown in Figure $4 \mathrm{~b}$. Moreover, the fields located at the bottom of metallic tips may be better than between two tips (induced by the VP laser), since the target nonmaterial is located at the bottom of AFM probes with high probability during the manipulation process, as Figure 1c,d shows. Hence, the electric field and trapping force around an NP should be investigated further. 

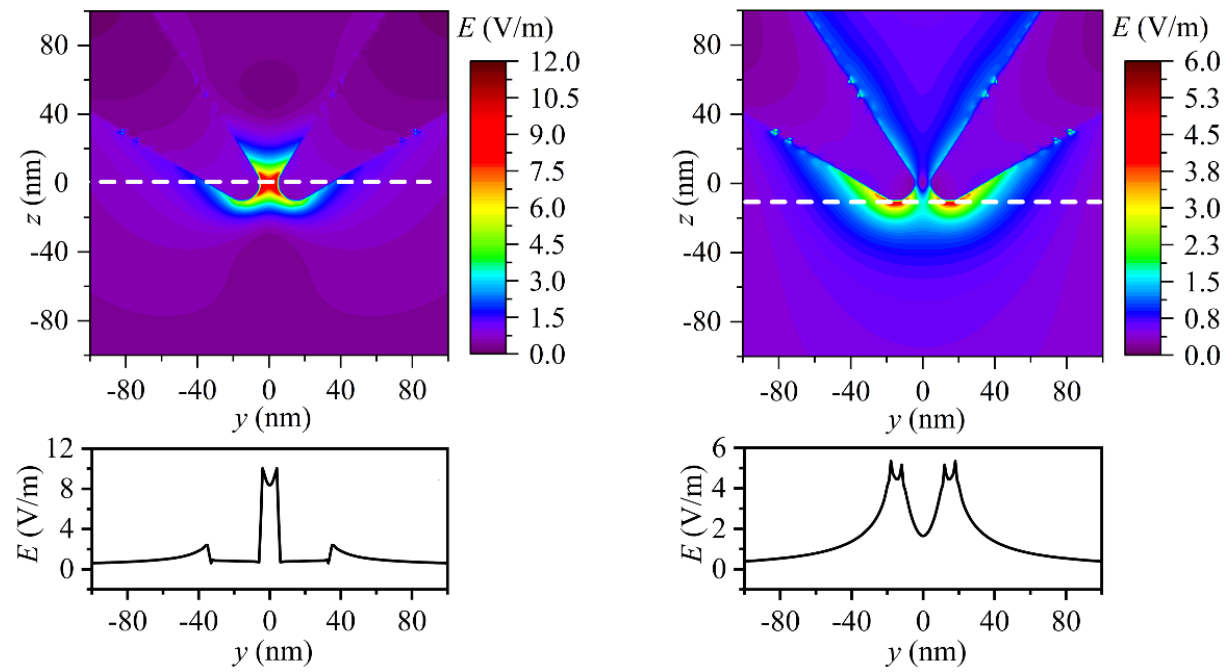

(a)

(b)
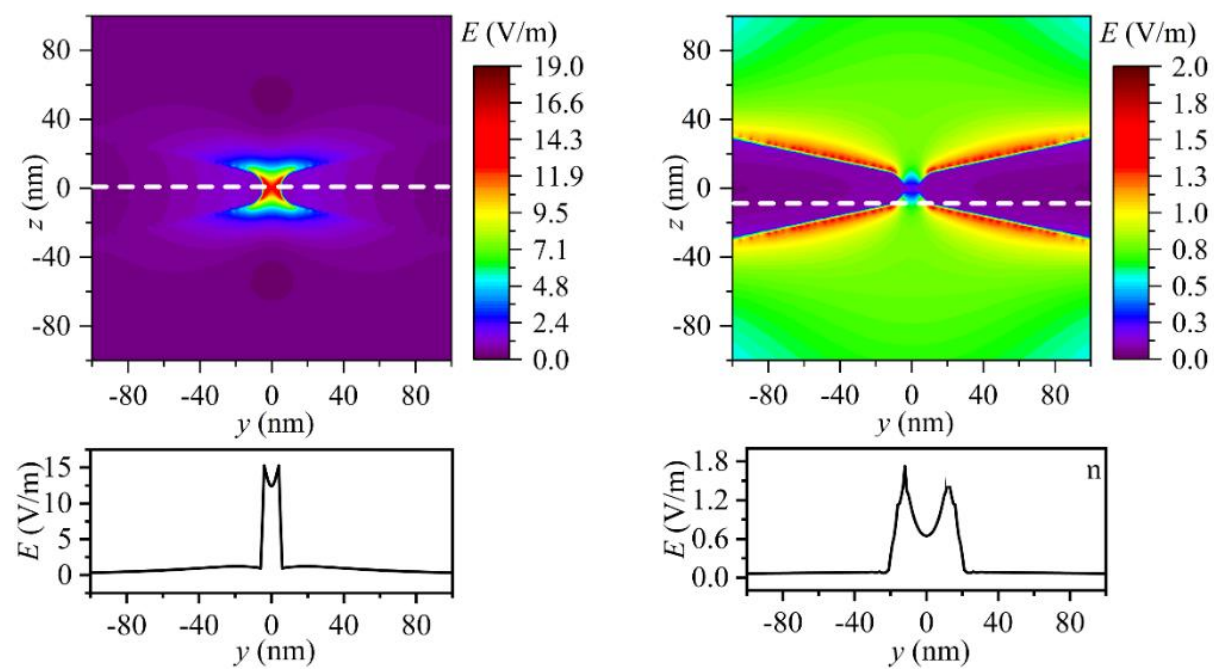

(c)

(d)

Figure 3. Distributions of electric fields in $y$ - $z$ plane (top diagram) and along $y$ direction (bottom diagram; the white dash lines in top diagram show the paths) around dual probe irradiated by polarized near-field laser. (a) ODPs irradiated by HP laser; (b) ODPs irradiated by VP laser; (c) CDPs irradiated by HP laser; (d) CDPs irradiated by VP laser.

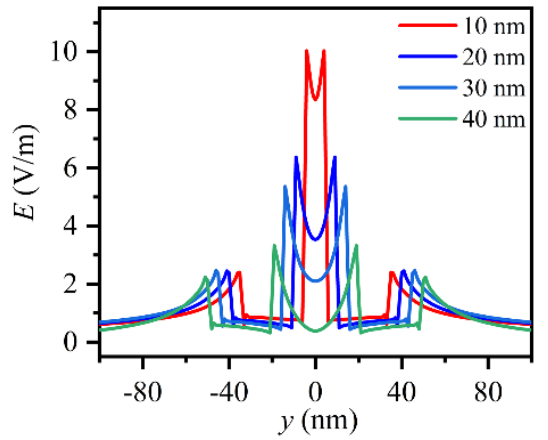

(a)

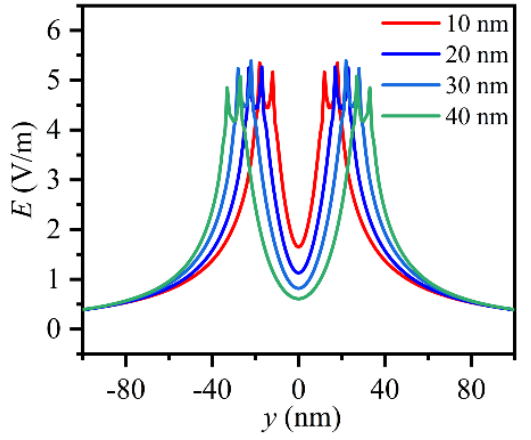

(b)

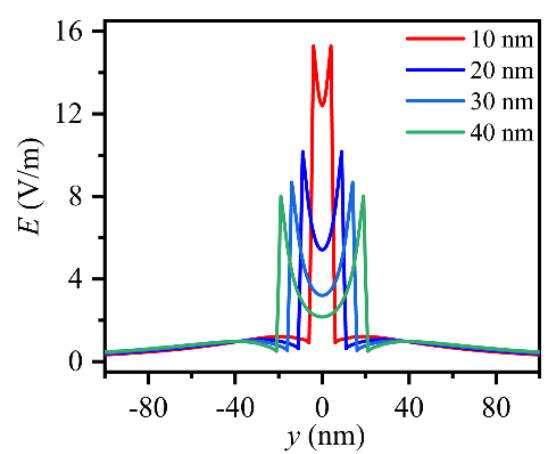

(c)

Figure 4. Distributions of electric field along $y$ direction around dual probe with different distances. (a) ODPs irradiated by HP laser; (b) ODPs irradiated by VP laser; (c) CDPs irradiated by HP laser. 


\subsection{Trapping Force of an NP in Enhanced Near-Field}

To reveal the manipulation ability of near-field induced by the combination of the SNOM probe and AFM probe, the electric field and trapping force exerted on an NP were calculated further. According to the above results, simulations of the single AFM probe irradiated by the VP laser from an SNOM probe were performed first. Figure 5a shows the distribution of the electric field when a silver NP with diameter $d=70 \mathrm{~nm}$ was added into the near-field (the distance between the probe tip and the NP was $D_{1}=10 \mathrm{~nm}$ ). Similar to the electric distribution of AFM probe without the NP, there is still an evanescent field between the AFM tip and the NP. However, the maximum intensity increases to $19.1 \mathrm{~V} / \mathrm{m}$, which is higher than the intensity without an NP. This strong enhancement with nanoscale size will apply an effective force on the silver NP. The intensity of the near-field at the end of the tip, as shown in Figure 1b, demonstrates that the suitable wavelength is still about $717 \mathrm{~nm}$ due to the strongest enhancement. Sequentially, based on the Maxwell stress tensor, total trapping force exerted by the near-field was calculated. Figure $5 c$ presents the total force induced by VP laser with wavelength in the range $300 \mathrm{~nm}-1200 \mathrm{~nm}$. The variations of optical force and electric intensity are highly correlated. So, it can be concluded again that evanescent fields with strong enhancement are the essential conditions for optical manipulation. The maximum force is about $8.2 \times 10^{-26} \mathrm{~N}$ (the value does not present the real value, due to the amplitude of the source laser being set to 1 ). To gain deeper insight into the orientation of the optical force, component forces along the $x, y, z$ axes were plotted in Figure 5d. For the laser wavelength from 600 to $1200 \mathrm{~nm}$, the component force along the $z$ axis is dominant, so that the evanescent field at the tip can trap the NP and move it toward the AFM probe. Optical force per unit volume also confirms this conclusion, as shown in Figure 5e. The red vectors, only at the top of the silver NP, mean that a large force along the $z$ direction is applied. However, in other directions, the disorder and the weak force are incapable of nano-manipulation, as the blue vectors show. So, the forces aroused by the near-field provide a possibility for the expected manipulation of an NP, while controlling the movement of the AFM and SNOM probes or the substrate. However, component force along the $x$ axis is significant in the wavelength range $300-600 \mathrm{~nm}$. This result can be attributed to forming the near-field around the aperture of the SNOM probe; see the distribution of the electric field for the $515 \mathrm{~nm}$ laser in Figure S3. These undesirable fields lead to disordered forces along the $x$ and $z$ directions, which will result in ineffective manipulation. So, avoiding the useless effect of the SNOM probe is also critical during manipulation. Then, the capacity of manipulation was evaluated further, varying particle size and the distance between the tip and NP. Figure S4 shows the total force versus wavelength. Here, Figure $6 \mathrm{a}, \mathrm{b}$ gives the total force as functions of diameter $d$ of an NP and the distance $D_{1}$, when the wavelength is $717 \mathrm{~nm}$. The optical force is linearly correlated with the diameter, which is similar to other optical trappings [29]. Since the effective area of the near-field is so small, the effect of diameter also reflects the edge shape of the nano-objects on manipulation. Apparently, the smaller curvature of the edge in the effective area causes higher enhancement and corresponding optical force, but the shape out of the effective area has limited influence. Figure S5 shows the larger trapping force of a nano-cube with a size of $70 \mathrm{~nm}$ greater than NPs, which confirms the conclusion. However, optical force decays exponentially as the distance increases. It is reasonable because the intensity of electric field also drops exponentially, as shown in Figure 2f. So, trapping ability is sensitively dependent on some critical factors, like the wavelength of the laser, the size and shape of objects, and the distance between probes and objects, which should be investigated further with experiments. 


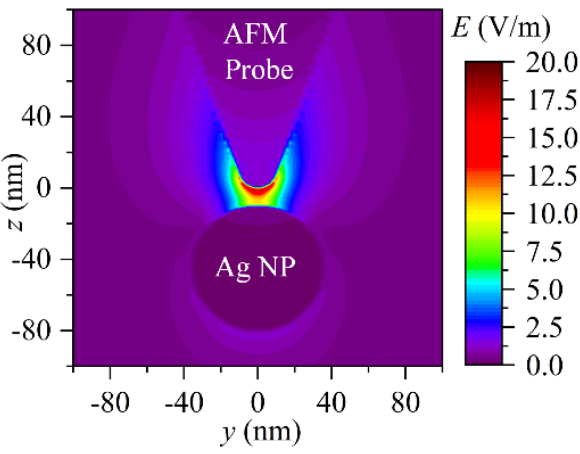

(a)

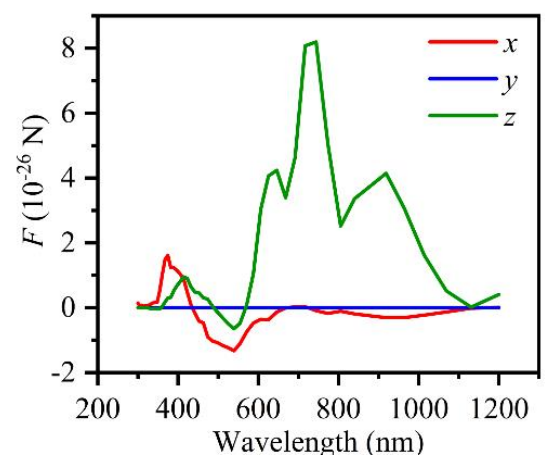

(d)

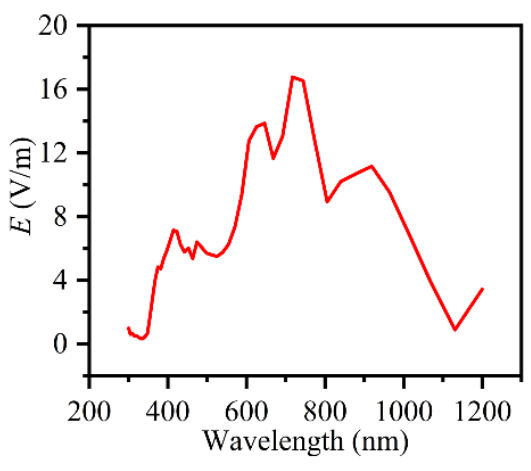

(b)

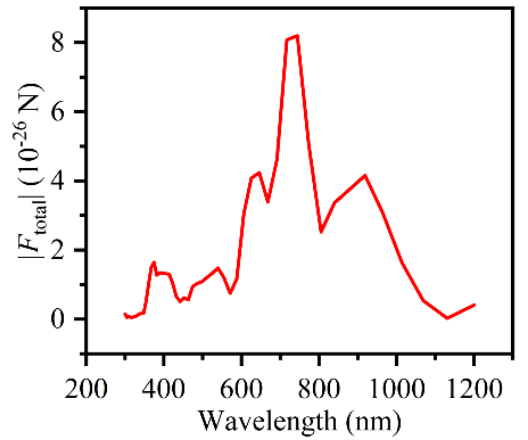

(c)

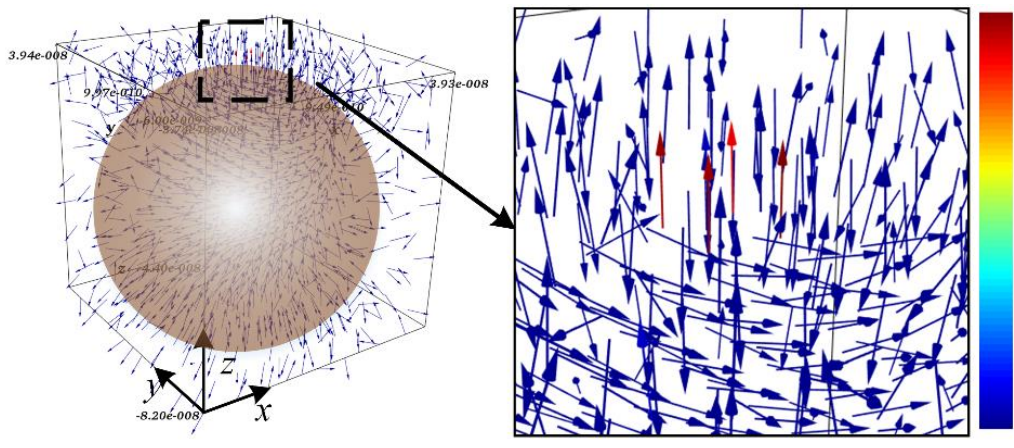

(e)

Figure 5. Trapping force of an NP using single probe irradiated by VP laser. (a) Distributions of electric field in $y$ - $z$ plane; (b) Electric intensities at the end of probe tip versus wavelength of incident laser; Total optical force (c) and component forces along $x, y, z$ axis (d) applied on the NP versus wavelength of incident laser; (e) Distributions of optical forces per unit volume in vector form for wavelength $717 \mathrm{~nm}$.

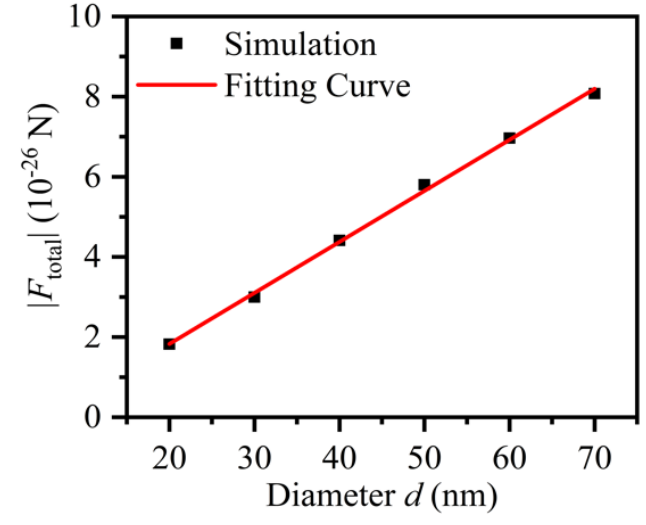

(a)

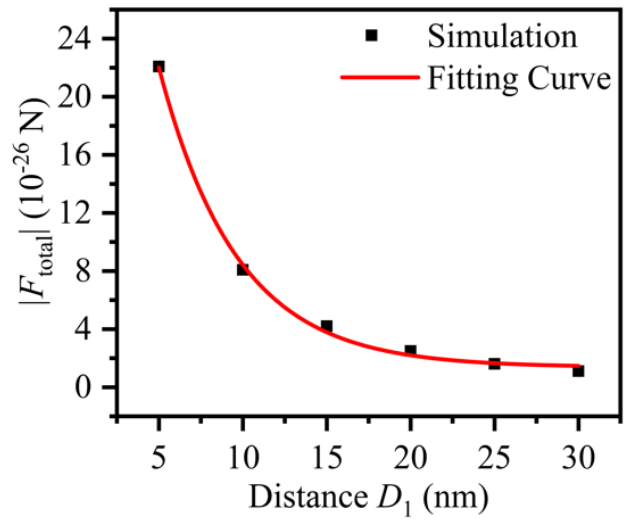

(b)

Figure 6. Total trapping force induced by VP laser with wavelength $717 \mathrm{~nm}$ as functions of some critical factors. (a) Diameter of NP; (b) Distance between the tip and the NP.

The simulations of trapping force with the single AFM probe have proven the feasibility of nano-manipulation using a polarized near-field laser. As a laser-irradiated dual probe can also arouse the effective electric-field, trapping forces of three configurations of the dual probe (ODPs irradiated by the HP laser, ODPs irradiated by the VP laser, and CDPs irradiated by the HP laser) were calculated to explore the excellent methods of enhancement. To make a comparison with the single probe, the distance between tips and NP was set to $D_{3}=10 \mathrm{~nm}$, as shown in Figure 1c. Figure 7 gives the component forces along $x, y, z$ axis applied on the silver NP versus the wavelength of the incident laser and corresponding 
distribution of electric field when the $z$ component forces are at their maximum. For the ODPs and CDPs irradiated by the HP laser, the max forces along the $z$ direction are about $2.6 \times 10^{-26} \mathrm{~N}$ and $3.0 \times 10^{-26} \mathrm{~N}$ respectively when the wavelengths are $964 \mathrm{~nm}$ and $744 \mathrm{~nm}$. These forces are even lower than the single probe irradiated by the VP laser, because the enhancement fields are located between two tips of AFM probes rather than between the tips and the NP, as the bottom diagrams of Figure 7a,c show. So, even the enhancement intensities of these two schemes with or without NP are high enough, but the trapping force also heavily depends on the location of the evanescent field. Figure $7 \mathrm{~b}$ presents a preferred manipulation scheme with maximum trapping force $\sim 11.3 \times 10^{-26} \mathrm{~N}$. Compared to the single probe irradiated by the VP laser (seeing Figure 5d), the dual probe has not only a higher trapping force, but also two suitable ranges of wavelengths around $717 \mathrm{~nm}$ and $646 \mathrm{~nm}$. If trapping forces along the $z$ direction larger than $7 \times 10^{-26} \mathrm{~N}$ were assumed to be effective for moving the NP, the suitable range wavelength of single probe would only be about $709-757 \mathrm{~nm}$, but $600-767 \mathrm{~nm}$ for dual probes. Such a wide wavelength range gives a more stable trap and also enormous potential in the aspect of application. The bottom diagram of Figure $7 \mathrm{~b}$ gives a deeper insight into the excellent manipulation capacity of the dual probe irradiated by the VP laser. The excellent manipulation ability can be attributed to the two metallic tips which provide a lager effect area of evanescent between the tips and the silver NP than the other two configurations. As the distance $D_{2}$ between tips and NP also has an effect on the trapping force, Figure S6 presents the trapping force and electric field of a dual probe with a narrow gap $\sim 5 \mathrm{~nm}$. The intensities of the optical force of all configurations increased as the distance decreases. No new effective peak of the force emerges, which means that the distance has little influence on the effective wavelength of the laser source for manipulation. Besides, a small gap can induce new evanescent fields between the tips and the NP for the dual probe irradiated by the HP laser, as the electric fields showed in Figure S6a,c. However, despite these new near-fields, compared to ODPs irradiated by the VP laser, the trapping forces of ODPs and CDPs irradiated by HP laser are still smaller. So, it is proven that the polarization direction of the incident laser and the configuration of two probes are dominant in optical manipulation, based on the combining scheme of the SNOM and AFM probes.
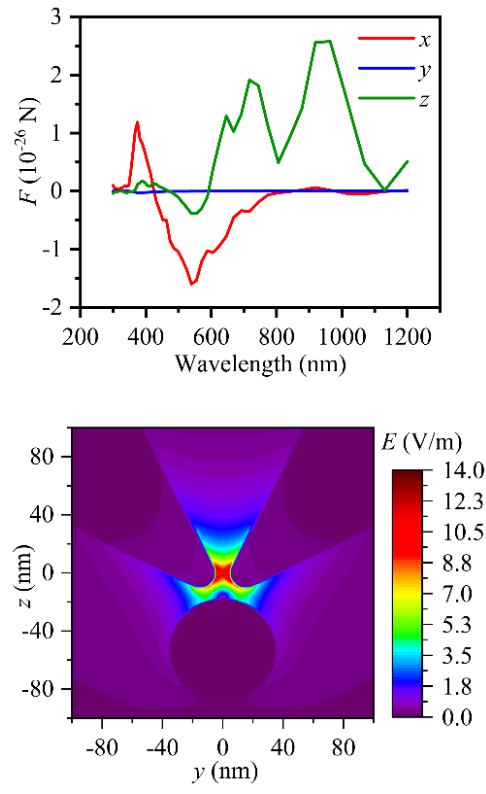

(a)
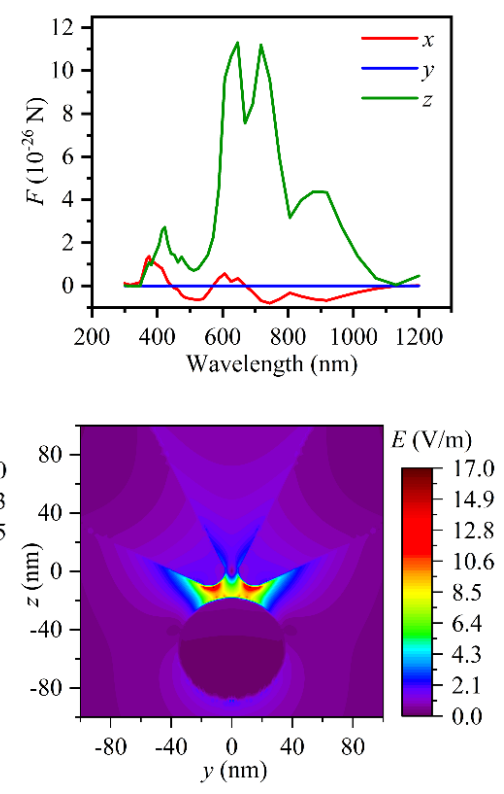

(b)
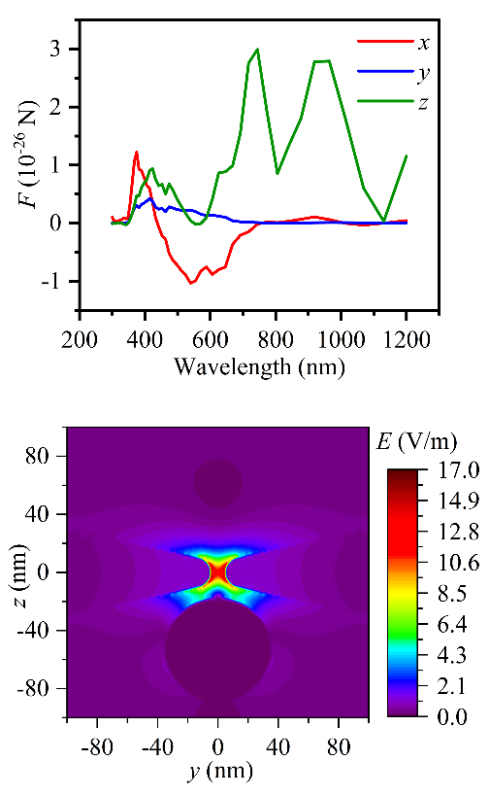

(c)

Figure 7. Component forces along $x, y, z$ axis applied on the NP versus wavelength (top diagram) and distributions of electric field in $y$ - $z$ plane (bottom diagram) around dual probe irradiated by polarized near-field laser. (a) ODPs irradiated by HP laser; (b) ODPs irradiated by VP laser; (c) CDPs irradiated by HP laser. 


\section{Conclusions}

This paper investigates the near-field and corresponding optical force around metallic single and dual probes irradiated by the polarized laser. The models of combing the AFM probe and SNOM probe were built and simulated based on the FDTD and Maxwell stress tensor. The distributions of electric fields around the tip of the single probe demonstrate that a polarized laser can induce nanoscale evanescent fields with high intensity, which are essential for optical manipulation. The intensity, area, and location of effective optical fields are mainly determined by the polarized direction of the laser and the configurations of probes. Moreover, near-field is easily aroused along the polarization direction at the surface of metallic probe, and the enhancement is higher if the angle between the polarization direction of the laser and the axis of the probe is smaller. Consequently, optical forces applied on an NP have proved that enhanced near-fields, in the condition of suitable parameters (e.g., wavelength, polarization direction, distance, configuration of probe), are capable of trapping the NP toward the tip. This means that NPs can be moved by probes with non-contact in the manipulation system. In addition, deeper insight into the dual probe scheme also shows that the different combinations of metallic probes and polarized lasers give a variety distribution of near-field and corresponding optical force. In particular, ODPs irradiated by VP laser exhibit higher trapping force and wider effective wavelength range than a single probe, which will improve the capacity of optical manipulating and expand the application of it. So, simulations in this paper provide a fertile library and are fundamental for realized promising manipulation by combining optical tweezers and probe manipulation.

Supplementary Materials: The following are available online at https:/ /www.mdpi.com/article/ 10.3390/app12020815/s1, Figure S1: Distributions of electric field in $y-z$ plane induced by polarized laser with different wavelengths, Figure S2. Distributions of electric field in $y-z$ plane for the configuration of ODPs with only one probe irradiated by HP laser with wavelengths $717 \mathrm{~nm}$, Figure S3: Distributions of electric field in $y-z$ plane induced by VP laser with wavelengths $511 \mathrm{~nm}$, Figure S4: The influence of diameter of NP and distance between the tip and the NP on the total optical force, Figure S5. Trapping force of a nano-cube using single probe irradiated by VP laser, Figure S6: Component forces along $x, y, z$ axis applied on the NP versus wavelength (top diagram) and distributions of electric field in $y-z$ plane (bottom diagram) around dual probe with distance $D_{2}=5 \mathrm{~nm}$ between tips and NP.

Author Contributions: Conceptualization, G.W. and Y.D.; methodology, G.W. and Y.D.; software, G.W., H.L. and X.L.; validation, G.W., Y.D. and H.L.; formal analysis G.W. and H.L.; investigation, G.W. and H.L.; resources, G.W. and H.L.; data curation, G.W.; writing-original draft preparation, G.W., Y.G. and H.L.; writing—review and editing, G.W. and Y.G.; visualization, G.W.; supervision, L.Y. and Y.W.; project administration, L.Y., Y.D. and Y.W.; funding acquisition, L.Y., Y.D. and Y.W. All authors have read and agreed to the published version of the manuscript.

Funding: This research was funded by National Key R\&D Program of China, grant number 2018YFB1107600; National Natural Science Foundation of China, grant number 52105437; Heilongjiang Provincial Postdoctoral Science Foundation, grant number LBH-Z20054; and China Scholarship Council, grant number 202006120184.

Institutional Review Board Statement: Not applicable.

Informed Consent Statement: Not applicable.

Data Availability Statement: Not applicable.

Acknowledgments: Genwang Wang acknowledges the supports given by A/P Kah-Wee Ang from National University of Singapore.

Conflicts of Interest: The authors declare no conflict of interest. 


\section{References}

1. Eigler, D.M.; Schweizer, E.K. Positioning single atoms with a scanning tunnelling microscope. Nature 1990, 344, 524-526. [CrossRef]

2. Jiang, C.; Lu, H.; Zhang, H.; Shen, Y.; Lu, Y. Recent advances on in situ SEM mechanical and electrical characterization of low-dimensional nanomaterials. Scanning 2017, 2017, 1-11.

3. Ono, M.; Kuramochi, E.; Zhang, G.; Sumikura, H.; Harada, Y.; Cox, D.; Notomi, M. Nanowire-nanoantenna coupled system fabricated by nanomanipulation. Opt. Express 2016, 24, 8647-8659. [CrossRef]

4. Ratchford, D.; Shafiei, F.; Kim, S.; Gray, S.K.; Li, X. Manipulating coupling between a single semiconductor quantum dot and single gold nanoparticle. Nano Lett. 2011, 11, 1049-1054. [CrossRef]

5. Wang, H.; Shi, Q.; Nakajima, M.; Takeuchi, M.; Chen, T.; Di, P.; Huang, Q.; Fukuda, T. Rail-guided multi-robot system for 3D cellular hydrogel assembly with coordinated nanomanipulation. Int. J. Adv. Robot. Syst. 2014, 11, 1-9. [CrossRef]

6. Vasić, B.; Matković, A.; Gajić, R.; Stanković, I. Wear properties of graphene edges probed by atomic force microscopy based lateral manipulation. Carbon 2016, 107, 723-732. [CrossRef]

7. Shi, C.; Luu, D.K.; Yang, Q.; Liu, J.; Chen, J.; Ru, C.; Xie, S.; Luo, J.; Ge, J.; Sun, Y. Recent advances in nanorobotic manipulation inside scanning electron microscopes. Microsyst. Nanoeng. 2016, 2, 1-16. [CrossRef]

8. Cao, C.; Zhang, Z.; Amirmaleki, M.; Tam, J.; Dou, W.; Filleter, T.; Sun, Y. Local strain mapping of GO nanosheets under in situ TEM tensile testing. Appl. Mater. Today 2019, 14, 102-107. [CrossRef]

9. Van der Lit, J.; Jacobse, P.H.; Vanmaekelbergh, D.; Swart, I. Bending and buckling of narrow armchair graphene nanoribbons via STM manipulation. New J. Phys. 2015, 17, 053013. [CrossRef]

10. Kim, S.; Ratchford, D.C.; Li, X. Atomic force microscope nanomanipulation with simultaneous visual guidance. ACS Nano 2009, 3 , 2989-2994. [CrossRef]

11. Lu, X.; Liu, J.; Ding, Y.; Yang, L.; Yang, Z.; Wang, Y. Simulation and fabrication of carbon nanotube-nanoparticle interconnected structures. Mech. Sci. 2021, 12, 451-459. [CrossRef]

12. Xie, H.; Haliyo, D.S.; Régnier, S. A versatile atomic force microscope for three-dimensional nanomanipulation and nanoassembly. Nanotechnology 2009, 20, 215301. [CrossRef]

13. Zimmermann, S.; Tiemerding, T.; Fatikow, S. Automated robotic manipulation of individual colloidal particles using vision-based control. IEEE/ASME Trans. Mechatron. 2014, 20, 2031-2038. [CrossRef]

14. Chen, H.; Zhang, X.L.; Zhang, Y.Y.; Wang, D.; Bao, D.L.; Que, Y.; Xiao, W.; Du, S.; Ouyang, M.; Pantelides, S.T.; et al. Atomically precise, custom-design origami graphene nanostructures. Science 2019, 365, 1036-1040. [CrossRef]

15. Moreno-Moreno, M.; Ares, P.; Moreno, C.; Zamora, F.; Gomez-Navarro, C.; Gomez-Herrero, J. AFM manipulation of gold nanowires to build electrical circuits. Nano Lett. 2019, 19, 5459-5468. [CrossRef]

16. Ashkin, A.; Dziedzic, J.M.; Bjorkholm, J.E.; Chu, S. Observation of a single-beam gradient force optical trap for dielectric particles Opt. Lett. 1986, 11, 288-290. [CrossRef]

17. Yan, Z.; Jureller, J.E.; Sweet, J.; Guffey, M.J.; Pelton, M.; Scherer, N.F. Three-dimensional optical trapping and manipulation of single silver nanowires. Nano Lett. 2012, 12, 5155-5161. [CrossRef]

18. Jauffred, L.; Taheri, S.M.R.; Schmitt, R.; Linke, H.; Oddershede, L.B. Optical trapping of gold nanoparticles in air. Nano Lett. 2015, 15, 4713-4719. [CrossRef] [PubMed]

19. Li, Y.; Guo, Z.; Qu, S. Living cell manipulation in a microfluidic device by femtosecond optical tweezers. Opt. Lasers Eng. 2014, 55, 150-154. [CrossRef]

20. Eriksson, E.; Sott, K.; Lundqvist, F.; Sveningsson, M.; Scrimgeour, J.; Hanstorp, D.; Goksör, M.; Granéli, A. A microfluidic device for reversible environmental changes around single cells using optical tweezers for cell selection and positioning. Lab A Chip 2010, 10, 617-625. [CrossRef]

21. Mandal, S.; Serey, X.; Erickson, D. Nanomanipulation using silicon photonic crystal resonators. Nano Lett. 2010, 10, 99-104. [CrossRef] [PubMed]

22. Zhang, W.; Huang, L.; Santschi, C.; Martin, O.J. Trapping and sensing $10 \mathrm{~nm}$ metal nanoparticles using plasmonic dipole antennas. Nano Lett. 2010, 10, 1006-1011. [CrossRef] [PubMed]

23. Kotnala, A.; Gordon, R. Quantification of high-efficiency trapping of nanoparticles in a double nanohole optical tweezer. Nano Lett. 2014, 14, 853-856. [CrossRef] [PubMed]

24. Keyi, W.; Zhen, J.; Wenhao, H. The possibility of trapping and manipulating a nanometer scale particle by the SNOM tip. Opt. Commun. 1998, 149, 38-42. [CrossRef]

25. Berthelot, J.; Aćimović, S.S.; Juan, M.L.; Kreuzer, M.P.; Renger, J.; Quidant, R. Three-dimensional manipulation with scanning near-field optical nanotweezers. Nat. Nanotechnol. 2014, 9, 295-299. [CrossRef] [PubMed]

26. Liu, B.; Yang, L.; Wang, Y. Optical trapping force combining an optical fiber probe and an AFM metallic probe. Opt. Express 2011, 19, 3703-3714. [CrossRef]

27. Liu, B.; Yang, L.; Wang, Y. Simulation of near-field optical manipulator using the combination of a near-field scanning optical microscope probe and an atomic force microscope metallic probe. J. Appl. Phys. 2011, 109, 104317. [CrossRef]

28. Cui, J.; Yang, L.; Wang, Y.; Mei, X.; Wang, W.; Hou, C. Nanospot soldering polystyrene nanoparticles with an optical fiber probe laser irradiating a metallic AFM probe based on the near-field enhancement effect. ACS Appl. Mater. Interfaces 2015, 7, 2294-2300. [CrossRef] [PubMed]

29. Liu, B.; Yang, L.; Wang, Y. Near-Field Optical Tweezers Based on NSOM and AFM Probes. Integr. Ferroelectr. 2012, 136, 59-65. [CrossRef] 